\title{
EMPREGO DE UM MODELO DE CRESCIMENTO E PRODUÇÃO PARA DETERMINAÇÃO DAROTAÇÃO EM POVOAMENTOS DE EUCALIPTO ${ }^{1}$
}

\author{
Robson Rodrigues Resende², Antonio Bartolomeu do Vale ${ }^{3}$, Thelma Shirlen Soares ${ }^{4}$, Márcio Lopes da Silva ${ }^{3}$, \\ Laércio Couto ${ }^{3}$ e Rodrigo Silva do Vale ${ }^{4}$
}

\begin{abstract}
RESUMO - O objetivo deste estudo foi realizar a prognose do crescimento e da produção a qualquer idade, associadas às informações de custo e receita, contemplando-se ciclos de um, dois e três cortes, bem como a determinação da idade técnica e econômica de corte. O critério para determinação da idade técnica de corte foi a maximização do incremento médio anual, enquanto a idade econômica de corte foi obtida com base no valor presente líquido, equiparando-se os horizontes no infinito para comparação das diferentes idades. Os resultados obtidos indicaram que as rotações técnica e econômica diferiram entre si e que ambas variaram em função do índice de local, portanto ao adotar uma rotação média por projeto pode haver perdas significativas de receita.
\end{abstract}

Palavras-chave: Prognose da produção, idade de corte e avaliação econômica.

\section{USE OF GROWTH AND YIELD MODEL FOR DETERMINATION OF ROTATION IN EUCALYPTUS STANDS}

\begin{abstract}
The objective of this study was to conduct a growth and yield projection of an eucalyptus stand at any age, associated to cost and revenue information, with cycles of one, two and three cuts, as well as to determine the technical and economic rotations. The criterion for determination of the technical age of cut was the maximization of the mean annual increment, while the economic age of cut was obtained based on the net present value, with the horizons being compared to the infinite for comparison of the different ages. The results indicated that the technical and economic rotations differed from each other with both varying in function of the site index; thus, adopting a medium rotation per project may cause significant revenue losses.
\end{abstract}

Key words: $\quad$ Yield projection, cutting age, economic evaluation.

\section{INTRODUÇÃO}

A previsão do crescimento de uma floresta, através de curvas de projeção do desenvolvimento em diâmetro, altura e volume, é a condição básica em qualquer processo de planejamento racional (Berger, 2000).

A determinação da idade de corte de um povoamento, também chamada de rotação florestal, é uma das fases mais importantes no conjunto das técnicas de manejo e planejamento florestal. No caso específico do empresário florestal, em que o objetivo principal é a maximização do lucro, esta fase reveste-se da maior importância, em razão do alto custo do empreendimento.

Uma empresa florestal pode utilizar as melhores técnicas de produção e plantio de mudas, usar a espécie mais adequada, atingir uma estrutura ótima de povoamento, adotar as técnicas de proteção e ainda ficar muito aquém dos resultados econômicos potencialmente

\footnotetext{
1 Recebido para publicação em 5.3.2002 e aceito para publicação em 30.4.2004.

2 Engenheiro Florestal. M.S. em Ciência Florestal, Duratex Florestal. ${ }^{3}$ Professor do Departamento de Engenharia Florestal da Universidade Federal de Viçosa - UFV, 36570-000 Viçosa-MG. ${ }^{4}$ Estudante de Doutorando em Ciência na UFV.
} 
atingíveis, caso o corte final e, ou, intermediário não for realizado no momento adequado (Bentley \& Teeguarden, 1965, citados por Resende, 1991).

A necessidade crescente do conhecimento da situação presente dos povoamentos, bem como de sua dinâmica, tem conduzido a um contínuo aprimoramento das técnicas de construção de modelos de crescimento e produção, os quais podem ser definidos como um conjunto de relações matemáticas que descrevem quantitativamente um sistema, representado neste caso pelo povoamento florestal. Além de informações sobre o estoque presente e suas mudanças com o tempo, é de interesse para o manejador conhecer o efeito da aplicação de técnicas silviculturais, como o controle do espaçamento, a intensidade de desbaste e a adubação (Campos et al., 1986).

Diante do exposto, os objetivos deste estudo foram estimar e avaliar, segundo critérios técnicos e econômicos, a idade de corte de um povoamento de eucalipto, empregando um modelo de crescimento e produção, bem como determinar a perda de receita ao adotar uma rotação média para o projeto.

\section{MATERIALEMÉTODOS}

\subsection{Fonte de Dados}

O estudo foi conduzido com dados provenientes de um povoamento de 253,2 ha de Eucalyptus grandis, plantado no espaçamento de 3,0 x 2,0 m, localizados na região de Martinho Campos-MG.

A idade média inicial considerada para projeção é de 30 meses em primeira rotação, considerando que os dados dendrométricos de input foram provenientes de parcelas medidas em povoamentos com essa idade.

O interesse deste estudo foi obter informações sobre as produtividades presente e futura da área, associadas a informações econômicas, e definir qual a estratégia de corte que permite operar próximo da idade de maximização do retorno líquido.

Para tanto foram efetuadas medições, em nível de parcela de inventário florestal, da circunferência a 1,30 m de altura do solo (cap) de todas as árvores e da altura de cinco árvores dominantes $(\mathrm{Hd})$.

\subsection{Modelo de Crescimento e Produção}

O conjunto de modelos de crescimento e produção utilizado para projeção da área basal e do volume corresponde ao proposto por Clutter (1963) para estudo de crescimento e produção em povoamentos de Pinus taeda. A forma particular do modelo utilizado compreende as seguintes equações:

- Índice de local:

$$
\ln (S)=\ln (H d)+\left(\frac{1}{I}-\frac{1}{I_{i}}\right) \times \ln \left(\beta_{1}\right)
$$

- Produção presente em volume (m³/ha):

$$
\ln (V)=\beta_{0}+\beta_{1} S+\beta_{2} I^{-1}+\beta_{3} \ln (B)
$$

- Projeção da produção em volume (m³/ha):

$$
\ln \left(V_{2}\right)=\beta_{0}+\beta_{1} S+\beta_{2} I_{2}^{-1}+\beta_{3}\left(\frac{I_{1}}{I_{2}}\right) \times \ln \left(B_{1}\right)+\beta_{4}\left(1-\frac{I_{1}}{I_{2}}\right)+\beta_{5} S\left(1-\frac{I_{1}}{I_{2}}\right)
$$

- Projeção da área basal $\left(\mathrm{m}^{2} \cdot \mathrm{ha}^{-1}\right)$ :

$$
\ln \left(B_{2}\right)=\left(\frac{I_{1}}{I_{2}}\right) \times \ln \left(B_{1}\right)+\alpha_{0}\left(1-\frac{I_{1}}{I_{2}}\right)+\alpha_{1} S\left(1-\frac{I_{1}}{I_{2}}\right)
$$

R. Árvore, Viçosa-MG, v.28, n.2, p.219-225, 2004 
- Crescimento corrente em área basal ( $\left.\mathrm{m}^{2} / \mathrm{ha} / \mathrm{ano}\right)$ :

$$
\frac{d B}{d I}=B I^{-1} \times \alpha_{0}+\alpha_{1} S-\ln (B)
$$

- Crescimento corrente em volume ( $\left.\mathrm{m}^{3} / \mathrm{ha} / \mathrm{ano}\right)$ :

$$
\frac{d V}{d I}=V\left[-\beta_{2} I^{2}+\beta_{3} B^{-1} \times\left(\frac{d B}{d I}\right)\right]
$$

em que $\mathrm{S}$ = índice de local; $\mathrm{I}$ = idade atual; $\mathrm{I}_{1}=$ idade inicial para projeção; $I_{2}=$ idade de projeção; $I_{i}=$ idade índice; $\mathrm{B}=$ área basal atual $\left(\mathrm{m}^{2}\right)$; $\mathrm{B}_{1}$ = área basal projetada na idade $\mathrm{I}_{1}\left(\mathrm{~m}^{2}\right) ; \mathrm{B}_{2}=$ área basal projetada na idade $\mathrm{I}_{2}\left(\mathrm{~m}^{2}\right)$; $\mathrm{V}=$ volume atual $\left(\mathrm{m}^{3}\right) ; \mathrm{V}_{2}=$ volume projetado na idade $\mathrm{I}_{2}$ $\left(\mathrm{m}^{3}\right)$; ln = logaritmo neperiano; e $\beta_{0}, \beta_{1}, \ldots, \beta_{5}, \alpha_{0}, \alpha_{1}=$ parâmetros a serem estimados.

Neste estudo, considerou-se que a produção do $2^{\circ}$ e do $3^{\circ}$ cortes corresponde a 90 e $80 \%$, respectivamente, em relação à produção do alto-fuste.

\subsection{Fluxo de Caixa}

As receitas, em um determinado tempo, foram oriundas do produto entre o volume de madeira no tempo $t$ pelo valor dessa madeira nesse mesmo tempo. A composição dos custos foi feita por seu período de ocorrência, considerando os componentes de custo apresentados no Quadro 1.

Para obtenção da receita bruta foram considerados, para madeira abatida e colocada disponível para transporte, os valores de US $\$ 9,00 / \mathrm{m}^{3}$ e de $6 \%$ a.a. para a taxa de juros.

\subsection{Avaliação Técnica}

O critério de avaliação técnica adotado visa a determinação da idade de corte do povoamento e tem como fator de estratificação o índice de local. Algebricamente, o critério pode ser definido da seguinte forma:

$$
\frac{I C A}{I M A}=1
$$

em que $I C A=$ incremento corrente anual ( $\left.\mathrm{m}^{3} / \mathrm{ha} / \mathrm{ano}\right)$; e $I M A=$ incremento médio anual ( $\left.\mathrm{m}^{3} / \mathrm{ha} / \mathrm{ano}\right)$.

A interpretação da expressão mostra que o ponto no tempo que igualar o valor do ICA ao IMA será a idade técnica de corte da floresta, ou seja, quando a razão entre os dois for igual a 1 .

\subsection{Avaliação Econômica}

Foram utilizados três critérios para análise de investimentos e, conseqüentemente, para determinação da idade ótima de corte do ponto de vista econômico. O critério de definição da idade economicamente ótima de corte utilizado foi o VPL (valor presente líquido). Adicionalmente, utilizou-se o BPE (benefício periódico equivalente) para ilustrar a rentabilidade periódica do investimento e o CMP (custo médio de produção) para obter uma estimativa do valor de produção da madeira. Nos casos do VPL e do CMP efetuou-se a equiparação dos horizontes no infinito.

Quadro 1 - Informações de custo das operações florestais a serem executadas na área

\begin{tabular}{|c|c|}
\hline Item de Custo & Valor (US\$/ha) \\
\hline \multicolumn{2}{|l|}{ Custo fixo de implantação } \\
\hline Derrubada da vegetação & 45,42 \\
\hline Destoca & 84,76 \\
\hline Enleiramento & 50,88 \\
\hline Desenleiramento & 10,42 \\
\hline Primeira gradagem & 45,31 \\
\hline Segunda gradagem & 13,96 \\
\hline Combate inicial à formiga* & 11,45 \\
\hline \multicolumn{2}{|l|}{ Custo variável de implantação } \\
\hline Mudas & 70,10 \\
\hline Adubação** & 53,88 \\
\hline Plantio & 23,39 \\
\hline Replantio & 8,44 \\
\hline \multicolumn{2}{|l|}{ Custos anuais } \\
\hline Combate à formiga* & 10,41 \\
\hline Conservação de estradas & 27,14 \\
\hline Conservação de aceiros & 7,99 \\
\hline \multicolumn{2}{|l|}{ Custos silviculturais } \\
\hline Capina & 23,29 \\
\hline Roçada & 11,76 \\
\hline Brotação & 5,62 \\
\hline Custo de colheita/m*** & 2,14 \\
\hline Valor da terra & 396,09 \\
\hline Custo administrativo & $15 \%$ dos custos totais \\
\hline
\end{tabular}

Table 1 - Information of the forest cost operations to be executed in the area

R. Árvore, Viçosa-MG, v.28, n.2, p.219-225, 2004 


\section{RESULTADOS E DISCUSSÃO}

\subsection{Estimativa da Rotação Técnica}

Com o objetivo de analisar a produção futura da área, executou-se um estudo da idade de corte dos talhões separadamente, o que permitiu definir a produção de cada sítio.

De acordo com os resultados obtidos (Quadro 2), observa-se uma diferença na idade de corte da floresta quando se considera a capacidade produtiva do local, aqui representada pelo índice de local. Podem ser observadas diferenças de até 11 meses entre duas capacidades produtivas, como ocorre com os índices de local 14 e 17.

Um resultado que merece atenção refere-se ao índice de local 17, que apresentou a menor idade técnica de corte, apesar de não ter a melhor produtividade. Isso é explicado pelo fato de a área basal inicial deste local ser superior à área basal inicial observada para o índice de local 18, por exemplo, que é o índice imediatamente superior em termos de capacidade produtiva.

A simples observação da função de projeção de volume futuro, em que a estimativa é obtida a partir de uma inter-relação entre idade atual, idade futura, índice de local e área basal, conforme equação 3, mostra a importância da área basal inicial nas estimativas feitas pelo modelo.

O efeito imediato desse tipo de relação pode ser representado pelo comportamento da sua curva de produção para os índices de local 17 e 18 (Figura 1a). Observa-se maior inclinação para a curva do índice de local 17 até aproximadamente 48 meses, sendo depois superada pela curva do índice de local 18. Esse fato leva a uma antecipação da idade técnica de corte (Figura 1b), evidenciando que a produção futura de uma floresta é uma inter-relação das suas características dendrométricas, havendo uma compensação em maior ou menor grau entre elas.

A expectativa de produção para o ciclo de três cortes é apresentada no Quadro 3, considerando que o intervalo de corte para cada índice de local é igual à rotação técnica estimada, e que a produção da primeira e a da segunda brotação sigam a premissa de queda de 10 e $20 \%$ em relação à produção observada para o alto fuste, respectivamente.

\subsection{Estimativa da Idade de Rotação Econômica}

A determinação da rotação econômica foi realizada, em nível de talhão, para um, dois e três cortes, sempre para horizonte infinito, com a inclusão do custo da terra, e analisando a receita obtida com a madeira colhida.

A importância de estudar três ciclos distintos de corte está na necessidade de se confrontar rentabilidade do empreendimento com substituição da floresta, uma vez que quanto menor o número de cortes maior a intensidade de novos plantios.

Os resultados obtidos (Quadro 4) mostram receita líquida negativa para os índices de local 14, 15, 17 e 18, quando se considera horizonte de um ou dois cortes, havendo redução do prejuízo quando se trabalha com o horizonte de dois cortes, devido à diluição dos custos de implantação num período de tempo maior, além da incorporação da receita da segunda rotação.

Os índices de local 19, 22 e 23 apresentaram receita líquida positiva para qualquer ciclo de corte considerado, havendo aumento da receita líquida para o índice de local 19, quando se aumentou o número de cortes. Analisando os índices 22 e 23, constata-se que houve aumento da

Quadro 2 - Estimativa da rotação técnica, em meses, por índice de local (S)

Table 2 - Technical rotation estimate, in months, per site index (S)

\begin{tabular}{|c|c|c|c|c|c|c|}
\hline $\begin{array}{c}\mathrm{S} \\
(\mathrm{m})\end{array}$ & $\begin{array}{c}\text { Área Basal Inicial } \\
\left(\mathrm{m}^{2} / \mathrm{ha}\right)\end{array}$ & Rotação Técnica & $\begin{array}{c}\text { Área } \\
\text { (ha) }\end{array}$ & $\begin{array}{l}\text { Volume } \\
\left(\mathrm{m}^{3} / \mathrm{ha}\right)\end{array}$ & $\begin{array}{c}\text { IMA } \\
\left(\mathrm{m}^{3} / \mathrm{ha} / \mathrm{ano}\right)\end{array}$ & $\begin{array}{c}\text { ICA } \\
\left(\mathrm{m}^{3} / \mathrm{ha} / \mathrm{ano}\right)\end{array}$ \\
\hline 14 & 5,38 & 59 & 12,5 & 61,8 & 12,6 & 13,2 \\
\hline 15 & 6,87 & 54 & 12,3 & 71,2 & 15,8 & 16,4 \\
\hline 17 & 9,44 & 48 & 25,6 & 91,0 & 22,8 & 23,7 \\
\hline 18 & 8,74 & 54 & 137,8 & 104,2 & 23,2 & 24,0 \\
\hline 19 & 9,76 & 53 & 12,9 & 118,4 & 26,8 & 27,7 \\
\hline 22 & 12,31 & 53 & 25,6 & 172,2 & 39,0 & 40,6 \\
\hline 23 & 14,70 & 50 & 25,6 & 197,4 & 47,4 & 48,8 \\
\hline
\end{tabular}

R. Árvore, Viçosa-MG, v.28, n.2, p.219-225, 2004 

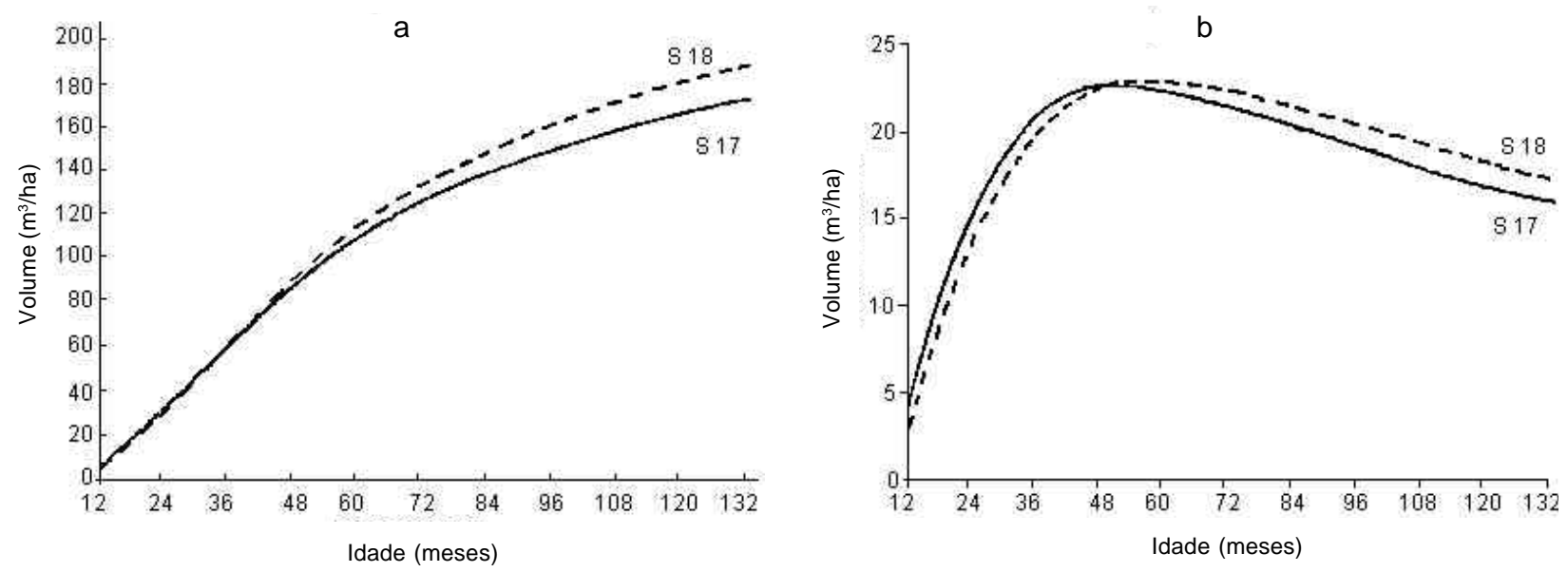

Figura 1 - Curvas de produção (a) e de crescimento médio anual (b) para os índices de local 17 e 18.

Figure 1 - Yield (a) and mean annual increment (b) curves for the site indexes 17 and 18.

receita líquida quando se passou do ciclo de um para o ciclo de dois cortes, mas o mesmo não ocorreu quando se considera o ciclo de três cortes, havendo ligeira diminuição da receita líquida. Este fato indica que os custos adicionais de se conduzir mais um ciclo não estão sendo compensados pelas receitas adicionais obtidas, promovendo uma equivalência em termos econômicos dos dois ciclos de corte.

Um aspecto importante a ser discutido é o fato de que a diminuição da idade de corte dentro de um único ciclo específico não aconteceu de forma proporcional ao aumento da capacidade produtiva do local. Uma das explicações seria que: quando da determinação da rotação técnica em nível de talhão, foi observada uma diferença máxima na idade técnica de corte, entre os locais de maior e menor índice de local igual a 9 meses. Esse tempo é

Quadro 3 - Expectativa de produção, em m³/ha por índice de local

Table 3 -Production expectation, in $m^{3 /}$ ha per site index

\begin{tabular}{|c|c|c|c|}
\hline Índice de Local & $1^{\underline{0}}$ Corte & $2^{\underline{0}}$ Corte & $3^{\mathbf{0}}$ Corte \\
\hline 14 & 61,8 & 55,6 & 49,4 \\
\hline 15 & 71,2 & 64,1 & 57,0 \\
\hline 17 & 91,0 & 81,9 & 72,8 \\
\hline 18 & 104,2 & 93,8 & 83,4 \\
\hline 19 & 118,4 & 106,6 & 94,7 \\
\hline 22 & 172,2 & 155,0 & 137,8 \\
\hline 23 & 197,4 & 177,7 & 157,9 \\
\hline
\end{tabular}

Quadro 4 - Estimativa da idade de rotação econômica (R), considerando uma idade inicial de 30 meses

Table 4 - Estimate of economic rotation age ( $R$ ), regarding an initial age of 30 months

\begin{tabular}{|c|c|c|c|c|}
\hline $\mathrm{S}^{*}$ & $\begin{array}{c}\mathrm{R} \\
\text { (meses) }\end{array}$ & $\begin{array}{c}\text { VPL } \\
\text { (US\$/ha) }\end{array}$ & $\begin{array}{c}\text { BPE } \\
\text { (US\$/ha/ano) }\end{array}$ & $\begin{array}{c}\mathrm{CMP} \\
\left(\mathrm{US} \$ \mathrm{~m}^{3}\right)\end{array}$ \\
\hline \multicolumn{5}{|c|}{ Ciclo de um corte } \\
\hline 14 & 84 & -1225 & $-73,5$ & 16,31 \\
\hline 15 & 72 & -968 & $-58,1$ & 13,39 \\
\hline 17 & 60 & -364 & $-21,8$ & 10,10 \\
\hline 18 & 60 & -298 & $-17,9$ & 9,87 \\
\hline 19 & 60 & 52 & 3,1 & 8,87 \\
\hline 22 & 60 & 1229 & 73,7 & 6,86 \\
\hline 23 & 60 & 2001 & 120,1 & 6,11 \\
\hline \multicolumn{5}{|c|}{ Ciclo de dois cortes } \\
\hline 14 & 72 & -995 & $-59,7$ & 14,82 \\
\hline 15 & 60 & -715 & $-42,9$ & 12,20 \\
\hline 17 & 60 & -101 & $-6,1$ & 9,32 \\
\hline 18 & 60 & -38 & $-2,3$ & 9,12 \\
\hline 19 & 60 & 297 & 17,8 & 8,21 \\
\hline 22 & 60 & 1424 & 85,4 & 6,41 \\
\hline 23 & 48 & 2234 & 134,0 & 5,76 \\
\hline \multicolumn{5}{|c|}{ Ciclo de três cortes } \\
\hline 14 & 60 & -930 & $-55,8$ & 14,44 \\
\hline 15 & 60 & -645 & $-38,7$ & 12,01 \\
\hline 17 & 48 & -32 & $-1,9$ & 9,10 \\
\hline 18 & 60 & 5 & 0,3 & 8,98 \\
\hline 19 & 60 & 327 & 19,6 & 8,10 \\
\hline 22 & 48 & 1419 & 85,1 & 6,37 \\
\hline 23 & 48 & 2205 & 132,3 & 5,66 \\
\hline
\end{tabular}

R. Árvore, Viçosa-MG, v.28, n.2, p.219-225, 2004 
menor do que aquele utilizado para se estimar os valores atualizados das receitas e de custos e, conseqüentemente, da idade de maximização do VPL, que é feita considerando a incidência desses valores anualmente, ou seja, em intervalos discretos de 12 meses. Deste modo, a sensibilidade desse critério de análise econômica é para múltiplos inteiros de 12. Uma maneira de se contornar esse problema seria trabalhar com capitalização contínua e aplicar técnicas de cálculo diferencial para obtenção do ponto de maximização do VPL.

\subsection{Análises Técnico-Econômicas}

Associada às estimativas volumétricas, foram efetuadas projeções da idade econômica de corte, para ciclo de três cortes, e calculada a perda de receita líquida para cada caso. O cálculo da perda de receita líquida, para situações em que a idade de corte é diferente da idade economicamente ótima, é feito por meio da diferença entre o VPL para as duas idades em questão.

Por se ter como objetivo executar os cortes próximos à idade de maximização do retorno líquido do investimento, procedeu-se à projeção para a idade de 60 meses de todos os talhões. Deste modo, é possível estimar a perda de rentabilidade e verificar se a quantidade de madeira disponível atende às necessidades de consumo.

Os resultados da projeção feita em torno dos 30 meses de idade estão no Quadro 5. Observa-se que perdas na receita líquida, em função da mudança na idade econômica de corte no caso dos índices de local 17, 22 e 23 , não ocorreram de forma proporcional às suas produtividades, ou seja, quanto maior o volume, maior a perda em se mudar a idade de corte. Tal fato é explicado pelo comportamento da curva de produção no índice de local 17, que por não ter uma relação crescente, quando comparado com os demais locais de área basal inicial e índice de local, apresenta perdas de receita líquida maiores que as apresentadas pelo índice de local 22.

Um perfil do fluxo de produção de madeira para a área estudada, levando-se em consideração os volumes totais produzidos em cada ano do ciclo de corte, está no Quadro 6, contemplando-se o corte de acordo com a idade técnica, econômica e efetiva de corte, no caso de 5 anos.

Os valores apresentados mostram menor produção de madeira para o ciclo técnico, porque as idades de corte nesse ciclo foram arredondadas para o ano mais próximo.

Quadro 6 - Perfil da produção de madeira, para ciclo de três cortes, com projeções a partir dos 30 meses de idade

Table 6 - Wood production profile, for a 3 cut cycle, with projections starting from 30 months of age

\begin{tabular}{|c|c|c|c|}
\hline \multirow{2}{*}{\begin{tabular}{c}
\multirow{2}{*}{$\begin{array}{c}\text { Idade } \\
\text { (anos) }\end{array}$} \\
\cline { 2 - 4 }
\end{tabular}} & $\begin{array}{c}\text { Técnico } \\
\left(\mathrm{m}^{3}\right)\end{array}$ & $\begin{array}{c}\text { Econômico } \\
\left(\mathrm{m}^{3}\right)\end{array}$ & $\begin{array}{c}\text { Efetivo } \\
\left(\mathrm{m}^{3}\right)\end{array}$ \\
\hline 4 & $28.553,3$ & $11.128,4$ & \\
\hline 5 & 772,5 & $19.396,0$ & $33.245,6$ \\
\hline 8 & $25.697,9$ & $10.014,7$ & \\
\hline 10 & 695,3 & 17462,6 & $29.929,8$ \\
\hline 12 & $22.842,6$ & $8.903,7$ & \\
\hline 15 & 618,0 & $15.516,6$ & $26.596,3$ \\
\hline Total & $79.179,6$ & $82.422,0$ & $89.771,7$ \\
\hline
\end{tabular}

Quadro 5 - Estimativa da produção, receita e rotação econômica, em meses, para corte aos 5 anos Table 5 - Estimate of production, revenue and economic rotation, in months, for cut at the 5 years

\begin{tabular}{|c|c|c|c|c|c|c|c|c|c|}
\hline \multirow{2}{*}{$\begin{array}{l}\mathrm{S}^{*} \\
\text { (m) }\end{array}$} & \multicolumn{3}{|c|}{ Volume (m³/ha) } & \multirow{2}{*}{$\begin{array}{l}\text { Área } \\
\text { (ha) }\end{array}$} & \multirow{2}{*}{ VPL** } & \multirow{2}{*}{ BPE*** } & \multirow{2}{*}{$\begin{array}{c}\mathrm{CPM} \\
\left(\mathrm{US} \$ / \mathrm{m}^{3}\right)\end{array}$} & \multirow{2}{*}{$\begin{array}{l}\text { Rotação } \\
\text { Econômica }\end{array}$} & \multirow{2}{*}{$\begin{array}{l}\text { Perda de } \\
\text { Receita** }^{* *}\end{array}$} \\
\hline & $\mathrm{I}$ & II & III & & & & & & \\
\hline 14 & 62,9 & 56,6 & 50,3 & 12,5 & -930 & $-55,8$ & 14,44 & 60 & 0,0 \\
\hline 15 & 78,9 & 71,0 & 63,1 & 12,3 & -645 & $-38,7$ & 12,01 & 60 & 0,0 \\
\hline 17 & 112,1 & 100,9 & 89,7 & 25,6 & -55 & $-3,3$ & 9,18 & 48 & $-23,0$ \\
\hline 18 & 115,5 & 104,0 & 92,4 & 137,8 & 5 & 0,3 & 8,98 & 60 & 0,0 \\
\hline 19 & 133,6 & 120,2 & 106,9 & 12,9 & 327 & 19,6 & 8,10 & 60 & 0,0 \\
\hline 22 & 194,5 & 175,1 & 155,6 & 25,6 & 1.409 & 84,5 & 6,33 & 48 & $-10,0$ \\
\hline 23 & 234,4 & 211,0 & 187,5 & 25,6 & 2.118 & 127,1 & 5,68 & 48 & $-87,0$ \\
\hline
\end{tabular}

* S = índice de local; ** valores expressos em US\$/ha; e *** valores expressos em US\$/ha/ano. 
A maior produção é alcançada com o ciclo efetivo de corte (5 anos), para todos os índices de local, devido ao fato de alguns locais indicarem idade econômica aos 4 anos, o que leva a uma produção menor do que aos 5 anos, quando se comparam estes resultados com o ciclo econômico.

Para definir qual o ciclo de corte ideal, deve-se ter em mente que essa decisão é função da rentabilidade esperada ou aceita para o investimento, da produtividade das rotações subseqüentes, do valor da madeira, da taxa de desconto e da estratégia de abastecimento adotada. A estratégia de abastecimento está fundamentalmente ligada ao planejamento a longo prazo, portanto, devido às características da cultura florestal (ciclo longo), a antecipação de informações torna-se de fundamental importância para definição da estratégia a ser adotada.

A escolha do melhor ciclo não pode ser feita sem se considerar a demanda de madeira, nem as características operacionais de exploração da área em questão. Deste modo, as estimativas devem ser sempre verificadas, para que possa estar operando dentro de limites aceitáveis de rentabilidade norteada por estimativas confiáveis de disponibilidade de madeira.

\section{CONCLUSÕES}

De acordo com os resultados obtidos, para as condições em que este estudo foi desenvolvido, concluise que:
- A rotação técnica apresenta menor produção de madeira, quando comparada com a rotação econômica.

- A maior produção é alcançada com rotação efetiva aos 5 anos, para todos os índices de local.

- Na rotação econômica, a lucratividade é maior quando se opera com ciclo de três cortes para os índices de local 14 a 19 e ciclo de dois cortes, para os índices 22 e 23.

- Na rotação técnica, a produção é maior no ciclo de um corte.

\section{REFERÊNCIAS BIBLIOGRÁFICAS}

BERGER, R. Crescimento e qualidade da madeira de um clone de Eucalyptus saligna Smith sob o efeito do espaçamento e da fertilização. 2000. 110 f. Dissertação (Mestrado em Engenharia Florestal) - Universidade Federal de Santa Maria, Santa Maria, 2000.

CAMPOS, J. C. C. et al. Aplicação de um modelo compatível de crescimento e produção de densidade variável em plantações de Eucalyptus grandis. Revista Árvore, v. 10, n. 2, p. 121-134, 1986.

CLUTTER, J. L. Compatible growth and yield models for loblolly pine. Forest Science, v. 9, n. 3, p. 354-371, 1963.

RESENDE, R. R. Emprego de um modelo de crescimento e produção para determinação da rotação em povoamentos de eucalipto. 1991. $81 \mathrm{f}$. Dissertação (Mestrado em Ciência Florestal) - Universidade Federal de Viçosa, Viçosa, 1991. 